\title{
The First National Report Card on Quality of Health Care in America
}

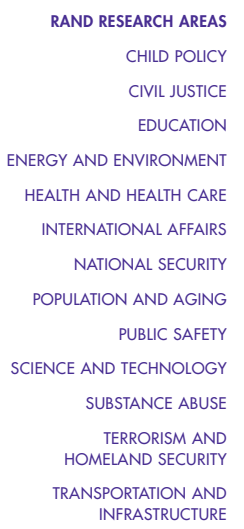

This product is part of the RAND Corporation research brief series. RAND research briefs present policy-oriented summaries of individual published, peer-reviewed documents or of a body of published work.

Corporate Headquarters 1700 Main Stree P.O. Box 2138 Santa Monica, California 90407-2138 Tel 310.393 .0411 Fax 310.393 .4818 (c) RAND 2004
$\mathrm{H}$ ow good is the quality of health care in America? To answer this question, Elizabeth McGlynn led a team of experts in the largest and most comprehensive examination ever conducted of health care quality in the United States. Called the Community Quality Index Study, it assessed the extent to which recommended care was provided to a representative sample of the U.S. population for a broad range of conditions in 12 metropolitan areas.

\section{Designing a National Report Card on Quality of Care}

The Community Quality Index Study differs from previous assessments of quality because it was more comprehensive, examined quality across the nation rather than in one geographic area, and included people with all types of insurance and a wide range of conditions. The research team used random telephone surveys to interview more than 13,000 adults in 12 metropolitan areas regarding their health care experiences (see Figure 1). About 6,700 individuals provided written consent for researchers to review their medical records and use the information to evaluate performance on 439 clinical indicators of quality for 30 acute and chronic

\section{Key findings:}

- Overall, adults received about half of recommended care.

- The level of performance was similar for chronic, acute, and preventive care.

- Quality of care varied substantially by condition.

- Performance was also similar in each of the metropolitan areas studied.

- No community had consistently the best or worst quality.

conditions, such as diabetes mellitus, asthma, hypertension (high blood pressure), and heart disease, and for related preventive care.

\section{Measuring Quality of Care}

To define and measure quality, the team developed a set of quality indicators, known as the RAND Quality Assessment (QA) Tools system. RAND staff selected 30 clinical areas representing the leading causes of death and disability as

\section{This Highlight summarizes RAND research reported in the following publications:}

McGlynn EA, Asch SM, Adams J, Keesey J, Hicks, J, DeCristofaro A, and Kerr EA, "The Quality of Health Care Delivered to Adults in the United States," New England Journal of Medicine, Vol. 348, No. 26, June 26, 2003, pp. 2635-2645.

Kerr EA, McGlynn EA, Adams J, Keesey J, and Asch SM, "Profiling the Quality of Care in Communities: Results from the Community Quality Index Study," Health Affairs, Vol. 23, No. 3, May/June 2004, pp. 247-256. 
Clinical Conditions Included in the RAND Quality Assessment Tools System

Alcohol dependence

Asthma

Atrial fibrillation

Benign prostatic hyperplasia

Breast cancer

Cancer pain and palliation

Cataracts

Cerebrovascular disease

Cesarean delivery

Chronic obstructive pulmonary disease

Colorectal cancer

Community-acquired pneumonia

Coronary artery disease

Depression

Diabetes mellitus

Dyspepsia and peptic ulcer disease

Headache

Heart failure

Hip fracture

Hyperlipidemia

Hypertension

Hysterectomy

Low back pain (acute)

Menopause management

Orthopedic conditions

Osteoarthritis

Prenatal care

Prostate cancer

Urinary tract infections

Vaginitis and sexually transmitted diseases

\section{Examples of Related Preventive Care}

Breast cancer screening

Cervical cancer screening

Colorectal cancer screening

Cigarette use counseling

Family planning/contraception

Immunizations

Screening for problem drinking

\section{Figure 1}

Metropolitan Areas in the Community Quality Index Study

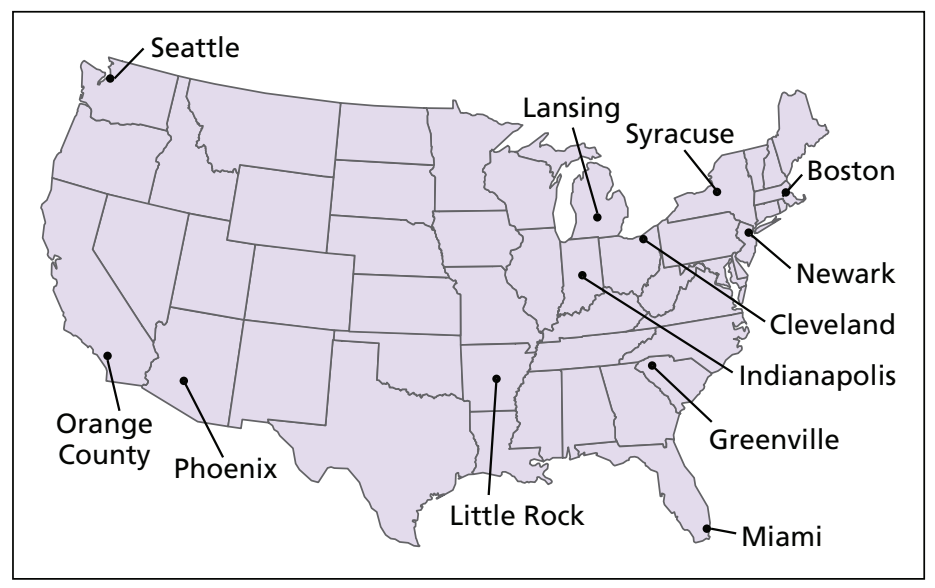

well as the major reasons that people seek care. They developed specific standards or quality indicators within each clinical area, based on a review of national guidelines and the medical literature. Panels of experts evaluated the proposed quality indicators. (All the quality indicators, including the literature reviewed and the panel recommendations, are available on the RAND Health web site at www.rand.org/health/ tools/qualist.html.) The researchers developed computerassisted medical record abstraction software to facilitate data collection by nurses.

The researchers constructed a quality "score" for each patient. The score was the number of times that the patient received the care recommended across all of the conditions that the patient had in a two-year period, divided by the number of times that the patient was determined to need specific health care interventions. The team then aggregated the individual scores to obtain quality scores for various dimensions of performance.

\section{Study Highlights}

- Overall, participants in the study received 55 percent of recommended care.

- Underuse of care was a greater problem than overuse. For example, patients failed to receive recommended care about 46 percent of the time, compared with 11 percent of the time when they received care that was not recommended and potentially harmful (see Figure 2).

- Overall performance was strikingly similar in all of the communities studied. Overall quality ranged from 59 percent in Seattle to 51 percent in Little Rock (see Figure 3). The researchers found the same basic level of performance for chronic, acute, and preventive care. 
- Quality varied substantially across conditions. For example, people with cataracts received about 79 percent of recommended care; persons with alcohol dependence received about 11 percent (see Figure 4).

- Preventive care. All communities did a better job of preventing chronic disease through screening tests (e.g., measuring blood pressure) and immunizations compared with other types of preventive care-for example, prevention of sexually transmitted diseases and HIV and counseling for substance abuse.

- Care for chronic disease. Care for selected chronic conditions (cardiac care, depression, hypertension, diabetes, and pulmonary problems) varied both across conditions and across communities within the same condition. For example: - Quality of care for hypertension was among the best for the chronic conditions-residents in Cleveland received about 70 percent of recommended care for this condition.

- Quality of care for cardiac conditions (including coronary artery disease, congestive heart failure, and atrial fibrillation) was generally lower than care for hypertension, ranging from 52 percent in Indianapolis and Orange County to 70 percent in Cleveland and Syracuse.

- Quality of care for pulmonary problems (asthma and chronic obstructive pulmonary disease) was lower than care for hypertension or cardiac conditions.

- Care for depression was even lower, ranging from 47 percent in Newark to 63 percent in Seattle.

- In most communities, quality of care for diabetes was lower than care for depression.

No single community had consistently the highest or lowest performance for all of the chronic conditions. The relative rankings of the communities changed depending on the aspect of care being examined. (See quality ratings for each community at http://www.rand.org/news/press.04/05.04table.pdf.)

\section{Does Poor Quality Matter?}

The deficits in care documented in the Community Quality Index Study pose serious threats to the health of the American public and translate into thousands of preventable complications and deaths per year (see the table on page 4).

- People with diabetes received only 45 percent of the care they needed. For example, less than one-quarter of diabetics had their average blood sugar levels measured regularly. Poor control of blood sugar can lead to kidney failure, blindness, and amputation of limbs.

- Patients with hypertension received less than 65 percent of recommended care. Poor blood pressure control is associated with increased risk for heart disease, stroke, and
Figure 2

Performance of the U.S. Health Care System

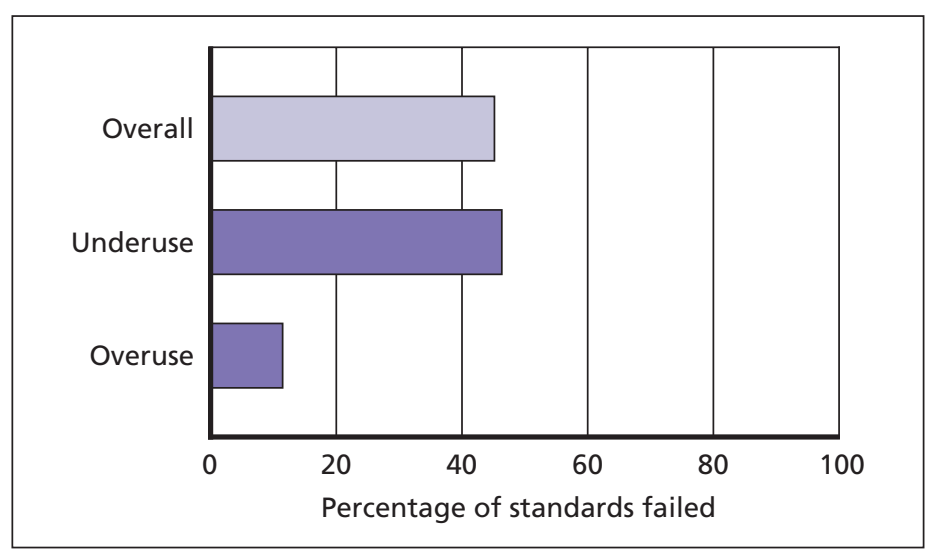

Figure 3

Quality of Care Was Similar in All the Communities Studied

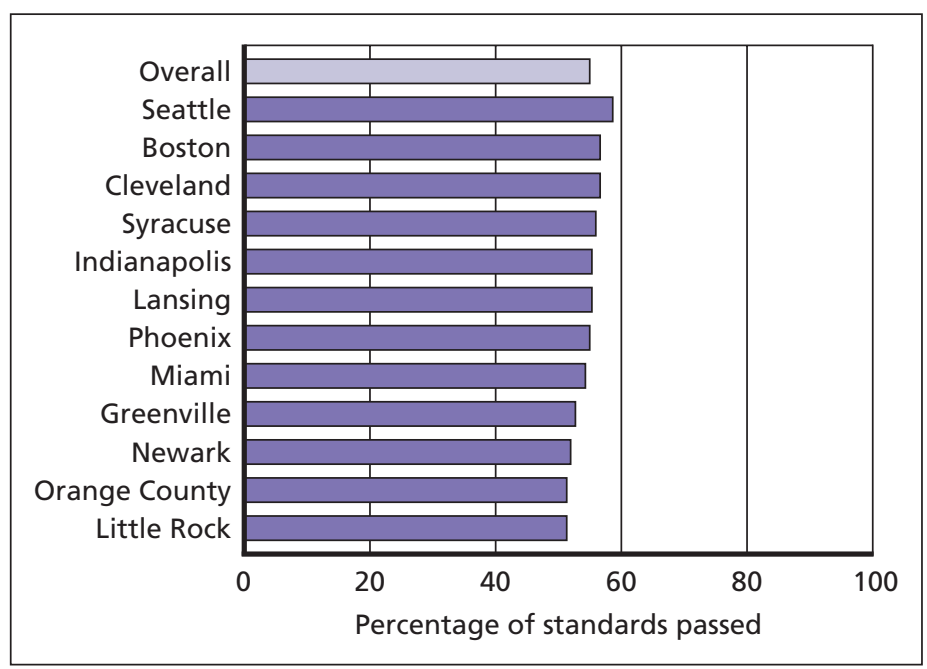

Figure 4

Management of Adults' General Medical Problems Varied Significantly

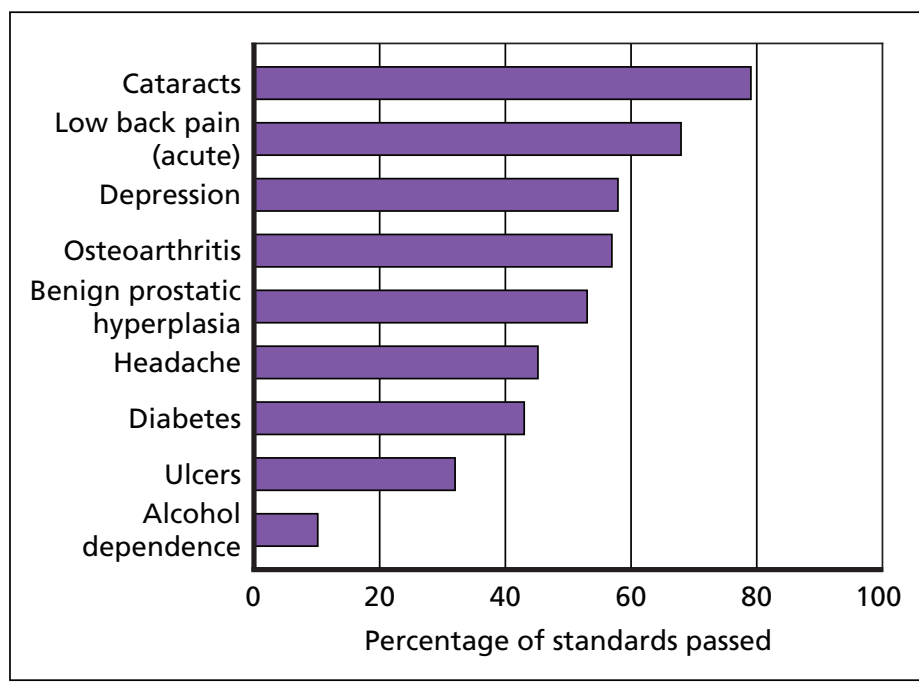


death. In fact, poor blood pressure control contributes to more than 68,000 preventable deaths annually.

- People with coronary artery disease received 68 percent of recommended care, but just 45 percent of heart attack patients received beta blockers and 61 percent got aspirinmedications that could reduce their risk of death by more than 20 percent.

- Patients with pneumonia received just 39 percent of recommended care. In fact, fewer than two-thirds of elderly Americans were vaccinated against pneumonia. Nearly 10,000 deaths from pneumonia could be prevented annually through proper vaccinations.

- Patients with colorectal cancer received 54 percent of recommended care, but just 38 percent of adults were screened for colorectal cancer. Routine tests and appropriate follow-up could prevent 9,600 deaths a year.

\section{What's Needed to Improve Care?}

This study provides the best estimates ever available about the quality of care in the United States. The study reveals substantial gaps between what clinicians know works and the care actually provided. These deficits persist despite initiatives by both the federal government and private health care delivery systems to improve care.

This study is not the first to identify poor-quality care. Studies stretching back over more than four decades have documented similar levels of poor performance. However, most people do not believe that there is a quality problem. Many think that the care delivered by their doctor, or in their community, is better than the care delivered in the nation as a whole.

What's needed to move the nation forward? The first step in improving care will be routine availability of information on health care performance at all levels. Information systems are needed that can produce the necessary measures of care routinely as part of the process of delivering care. Achieving this will require a major overhaul of our current health information systems, with a focus on automating the entry and retrieval of key data to support clinical decisionmaking and to measure and report quality.

Information is essential, but it isn't enough. We need to create teams of health professionals - doctors, nurses, and health educators-who work together to give each individual the care needed. Those teams must have tools to help them quickly identify the best set of services for each patient and be able to explain the choices clearly so that patients can choose the treatment that is right for them.

Communities need to examine their quality of care and determine how they might approach local quality initiatives. Large employers could provide leadership to improve chronic disease care in outpatient settings. Health care systems could collaborate with communities to improve preventive care.

Perhaps most important, patients must take responsibility for their own care. They should seek information from trusted sources - such as their physicians, health care agencies specializing in their condition or disease (e.g., the American Diabetes Association), and organizations specializing in preventive care (e.g., the U.S. Preventive Services Task Force)—to learn what kind of preventive care or treatment they should be receiving, then work with their physicians to ensure that they get recommended care. Patients should not assume that their physicians will remember all that needs to be done. They can help their physicians provide good care by being active advocates for it.

\section{Why Quality Standards Matter}

\begin{tabular}{|l|l|l|}
\hline Condition & \multicolumn{1}{|c|}{ What We Found } & Potentially Preventable Complications or Deaths (annual) \\
\hline Diabetes & Average blood sugar not measured for $24 \%$ & 2,600 blind; 29,000 kidney failure \\
Hypertension* & Less than $65 \%$ received indicated care & 68,000 deaths \\
Heart attacks* & $39-55 \%$ did not receive needed medications & 37,000 deaths \\
Pneumonia* & $36 \%$ of elderly received no vaccine & 10,000 deaths \\
Colorectal cancer* & $62 \%$ not screened & 9,600 deaths \\
\hline
\end{tabular}

*Source: Woolf SH, "The Need for Perspective in Evidence-Based Medicine," Journal of the American Medical Association, Vol. 282, 1999, pp. 2358-2365.

\footnotetext{
Abstracts of all RAND Health publications and full text of many research documents can be found on the RAND Health web site at www.rand.org/health. The RAND Corporation is a nonprofit research organization providing objective analysis and effective solutions that address the challenges facing the public and private sectors around the world. RAND's publications do not necessarily reflect the opinions of its research clients and sponsors. RAND ${ }^{\circledR}$ is a registered trademark.
}

RAND Offices Santa Monica - Washington - Pittsburgh - New York - Doha - Berlin - Cambridge - Leiden 
CHILD POLICY

CIVIL JUSTICE

EDUCATION

ENERGY AND ENVIRONMENT

HEALTH AND HEALTH CARE

INTERNATIONAL AFFAIRS

NATIONAL SECURITY

POPULATION AND AGING

PUBLIC SAFETY

SCIENCE AND TECHNOLOGY

SUBSTANCE ABUSE

TERRORISM AND HOMELAND SECURITY

TRANSPORTATION AND INFRASTRUCTURE
This PDF document was made available from www.rand.org as a public service of the RAND Corporation.

This product is part of the RAND Corporation research brief series. RAND research briefs present policy-oriented summaries of individual published, peerreviewed documents or of a body of published work.

The RAND Corporation is a nonprofit research organization providing objective analysis and effective solutions that address the challenges facing the public and private sectors around the world.

\section{Support RAND}

Browse Books \& Publications

Make a charitable contribution

\section{For More Information}

\author{
Visit RAND at www.rand.org \\ Explore RAND Health \\ View document details
}

\section{Limited Electronic Distribution Rights}

This document and trademark(s) contained herein are protected by law as indicated in a notice appearing later in this work. This electronic representation of RAND intellectual property is provided for non-commercial use only. Permission is required from RAND to reproduce, or reuse in another form, any of our research documents for commercial use. 